\title{
Functions of the Heterologous Intron-Derived Fragments Intra and Extra Factor IX-cDNA Coding Region on the Human Factor IX Expression in HepG2 and Hek-293T Cells
}

\author{
Mohammad Reza Sam ${ }^{1 *}$, Alireza Zomorodipour ${ }^{2}$, Aliakbar Haddad-Mashadrizeh ${ }^{3}$, Mahdi Ghorbani ${ }^{2}$, \\ Gholam Ali Kardar ${ }^{4}$, Sohrab Sam ${ }^{1}$ \\ ${ }^{1}$ Department of Cellular and Molecular Biotechnology, Institute of Biotechnology, Urmia University, Urmia, Iran \\ ${ }^{2}$ Department of Molecular Medicine, Institute of Medical Biotechnology, National Institute of Genetic Engineering and Biotechnology, \\ Tehran, Iran \\ ${ }^{3}$ Department of Biology, Faculty of Science, Ferdowsi University of Mashhad, Mashhad, Iran \\ ${ }^{4}$ Immunology, Asthma and Allergy Research Institute, Tehran University of Medical Sciences, Tehran, Iran
}

"Corresponding author: Mohammad Reza Sam, Department of Cellular and Molecular Biotechnology, Institute of Biotechnology, Urmia University, Urmia, Iran. P. O. Box: 165. Tel/Fax:+98-4433440199, E-mail: m.sam@urmia.ac.ir

Received: 18 Nov. 2016; $\quad$ Revised: 3 Jan. 2018; $\quad$ Accepted: 13 Jan. 2018; $\quad$ Published online: 15 May 2018

\begin{abstract}
Background: Human FIX (hFIX) gene transfer into hepatocytes has provided a novel approach for treatment of hemophilia B. To obtain an improved expression of hFIX, the functional $h F I X$-expressing plasmids with appropriate intron-derived fragments which facilitate transcription and promote an efficient 3 '-end formation of mRNAs are required.

Objectives: We aim to evaluate the functions of the heterologous intron-derived fragments intra and extra hFIX-cDNA coding region with respect to the hFIX expression in the hepatocytes and kidney cells.

Materials and Methods: HepG2 cells as differentiated hepatocytes and Hek-293T cells as embryonic kidney cells were transfected with the different $h F I X$-expressing plasmids containing various combinations of the two human beta-globin $(h B G)$ introns within the $h F I X$ cDNA and Kozak sequence. In the next stage, as a hepatocyte-specific sequence, the rat aldolase B intronic enhancer sequence ( $r A B E)$, was isolated from the first intron of the rat aldoase B gene and inserted within the upstream CMV promoter (CMVp) and efficacies of the engineered vectors were investigated in the stably-transfected HepG2 cells.

Results: Our data indicate that the intron-less construct and $h B G$ intron-I containing construct are more effective with regard to hFIX expression compared to other constructs in Hek-293 cells. In HepG2 cells, the rABE in combination with CMVp in context of intron-less plasmid induced an increase in total expression of hFIX protein dramatically; ranging from 2.3 to 40 folds increase compared to other constructs. The $r A B \mathrm{E}$ in combination with CMVp in the $h B G$ intron-I, $h B G$ intron-II, and $h B G$ intron-I,II containing plasmids induced 3.7, 2, and 1.6-fold increase in the total expression of hFIX protein, respectively. The presence of both $h B G$ intronic sequences within the $h F I X$-cDNA induced a higher secretion level of hFIX than either intron-I or II alone and provided correctly spliced hFIX transcripts in HepG2 and kidney cell lines. The intron-less construct with or without $r A B \mathrm{E}$ induced the highest hFIX mRNA levels in HepG2 and Hek-293T cells respectively compared to other constructs.

Conclusions: The embryonic kidney cells in addition to the differentiated hepatic cell lines could be successfully targeted by plasmid vectors. The intron-less and $h B G$ intron-I containing plasmids represent a particular interest in producing recombinant hFIX in Hek-293T cells. The synergistic function on the hFIX expression that was achieved by combining the CMVp with the liver-specific $r A B E$ would be a useful approach for future designing of the expression cassettes for hepatocyte-mediated gene expression in hemophilia B.

Keywords: Aldolase B intronic enhancer, Beta-globin introns, Hepatocyte, Hemophilia B, Human factor IX (hFIX), Plasmid
\end{abstract}

\section{Background}

The deficiency in the human factor IX (hFIX) results in the inherited bleeding disorder; which is known as the hemophilia B (1). The current protein-based therapy has improved the life expectancy in hemophilia B patients, but contamination of blood supply with blood-borne infectious agents, and the cost of therapy with the recombinant hFIX as well as development of neutralizing antibodies in patients plasma have fueled interests in cell-based gene therapy of Christmas disease (2-5).

Copyright (C) 2017 The Author(s); Published by National Institute of Genetic Engineering and Biotechnology. This is an open access article, distributed under the terms of the Creative Commons Attribution-NonCommercial 4.0 International License (http://creativecommons.org/licenses/ by-nc/4.0/) which permits others to copy and redistribute material just in noncommercial usages, provided the original work is properly cited. 
Human FIX gene transfer into the cells has provided a novel approach for treatment of hemophilias. In this regard, kind of target cells, low level of hFIX expression, and formation of neutralizing antibodies against viral vector and hFIX protein are the major challenges that need attention.

A number of cell types have been targeted for the somatic gene therapy of hemophilia B, which include bone marrow mesenchymal stem cells $(6,7)$, keratinocytes (8), myoblasts (9), hematopoietic stem cells (3), endothelial cells (10), and hepatocytes $(5,11)$. As liver is the major organ, responsible for expression of hFIX and is able to induce antigen-specific tolerance (12), hepatocytes represent excellent cells for the hFIX gene transfer.

It has been shown that the presence of an intron may increase the expression efficiency of the therapeutic proteins in human expression systems, at various post transcriptional processes $(13,14)$. In this context, it has also been shown that gene expression is dependent on the presence of introns and varies with the number of introns included (15) and intronic sequences function synergistically to increase the mRNA level, an effect that would requires the presence of two introns at least (16). Related to this issue, the enhancer-like activities of the human beta-globin $(h B G)$ introns (I, II) on the hFIX expression have been documented (17). It has been also shown that, introduction of the $h B G$ intron I into the identical position in the dehydofolate reductase cDNA has resulted to an enhanced expression of dehydofolatereductase protein more than that of native intron I of the DHFR gene in vitro (18).

The use of strong enhancer may add a positive effect on the gene expression. In our bioinformatic analysis on the first intron of the rat aldolase $\mathrm{B}(r A B)$ gene revealed the potential of enhancer-like activity of the region (+1916 to $+2329 \mathrm{nt})$ in hepatocytes. Therefore, in our manipulation strategy, different hFIX minigenes were constructed in which heterologous $h B G$ introns (I and II) were inserted in their corresponding sites within the $h F I X$-cDNA and a Kozak element were introduced upstream the hFIX initiation codon. In the next stage, rat aldolase $\mathrm{B}$ intronic enhancer $(r A B \mathrm{E})$ was inserted upstream of the CMV promoter (CMVp) in plasmids and efficacies of the engineered vectors were evaluated in the stably-transfected HepG2 cells.

\section{Objectives}

In the current research, we aim to evaluate the functions of human $\beta$-globin introns (I and II) within and outside of the coding region of the hFIX-cDNA on the expression of hFIX in HepG2 and Hek-293T cells.

\section{Materials and Methods}

\subsection{Cell lines, Constructs, and Primers}

HepG2 cell line (NCBI-C158) as human differentiated hepatocytes (19) and Hek-293T cells (NCBI-C644) as embryonic kidney cells were obtained from the National Cell Bank of Iran (Tehran, Iran). RPMI-1640 powder was purchased from Gibco, Germany. ELISA kits for the measuring concentration of the hFIX protein, human plasma depleted of FIX, and aPTT reagent were obtained from Diagnostica Stago (Asni eressur Seine, France). TA cloning kit was purchased from Fermentas, Canada. pET26-hFIX was used as the source of $h F I X$ cDNA (17). T4 DNA ligase was purchased from Roche, Germany. Plasmid pcDNA3.1 was purchased from Invitrogen, Carlsbad, CA, USA. Oligonucleotides were made by MWG Biotech (Ebersberg, Germany).

\subsection{Bioinformatic Analysis and Amplification of the $r A B E$}

The $r A B E$ nucleotide sequence was retrieved from Ensembl (ENSG00000136872). The existence of the regulatory motifs in the nucleotide sequence was investigated using MatInspector program (http://www. genomatix.de).

To amplify the enhancer sequence $(+1916$ to $+2329 \mathrm{nt})$ of the $r A B$ gene, DNA was isolated from the peripheral blood of Rattus Norvegicus and was subjected to the PCR method with $r A B$ E specific primers (Table 1).

To insert the PCR products into the pcDNA3 vector, $B g l \mathrm{II}$ and $\mathrm{MunI}$ sites were introduced at the 5 '-ends of the forward and reverse primers, respectively (20).

\subsection{Construction of the Bioengineered Vectors}

A 320 bp of the $h F I X$-cDNA 5'-end was amplified, using primer pairs hKozF9-F (carrying Kozak element, GCCACC) and hFIXE4-R (Table 1) (17) containing BamHI and DraI sites at their 5'-ends respectively (20). Thereafter, PCR was performed according to the standard protocol.

After cloning into a pTZ57R/T vector, the $320 \mathrm{bp}$ fragment from the hFIX-cDNA 5 '-end was used to substitute its corresponding fragment in the plasmid pET26-hFIX between BamHI and DraI sites. Subsequently, the Kozak-containing $h F I X$-cDNA was sub-cloned between BamHI and NotI sites in the pcDNA3.1. The generated construct was named pCMV. FIX (Fig. 1).

To introduce $r A B E$ upstream the CMVp, the amplified $r A B \mathrm{E}$ was inserted between $B g l \mathrm{II}$ and $M u n \mathrm{I}$ sites in the plasmid, pCMV.FIX to construct plasmid $\mathrm{p} r A B \mathrm{E} /$ CMV.FIX (Fig. 1). In the next step, the $r A B E / C M V p$ 
Table 1. List of primers used for the construction of hFIX expression cassettes.

\begin{tabular}{lll}
\hline Primer name & Nucleotide sequence $\left(5^{\prime}\right.$ to $\left.3^{\prime}\right)$ & Restriction site \\
\hline rABE-1912F & GAAGATCTAGGCCTCCACACTCTATTGCA & BglII \\
rABE-2329R & AGCAATTGGTCAAGTCAAACTCGTCCTGT & MunI \\
hKozF9-F & GGATCCGCCACCATGCAGCGCGTGAACATGAT & BamHI \\
hFIXE4-R & CC & DraI \\
hFIX-R2 & GAAGCTTTCTCCCTTTGTGGAAGACTCTTCCC & HindIII \\
$\beta$ actin-F & GAGACCTTCAACACCCCAGCC & - \\
$\beta$ actin-R & AGACGCAGGATGGCATGGG & - \\
\hline
\end{tabular}

Restriction sites are underlined. The Kozak sequence is boxed.

fragment from the prABE/CMV.FIX was isolated and used to substitute the CMVp between BglII and HindIII sites in the plasmids named as pCMV.FIX-I (containing $h B G$ intron-I), pCMV.FIX-II (containing $h B G$ intronII) and pCMV.FIX-I,II (containing $h B G$ intron-I, II) to generate new plasmids named as named as $\mathrm{p} r A B \mathrm{E} /$ CMV.FIX-I, prABE/CMV.FIX-II, and prABE/CMV. FIX-I, II, respectively (Fig. 1). The procedure for the construction of the current vectors has been described in our previous study in detail (17).

\subsection{Transfection of hFIX-Expressing Plasmids}

Transfection of the $h F I X$-expressing plasmids into HepG2 cells was described earlier in detail (20). 48 hours post transfection, the conditioned medium was collected for evaluation of hFIX biological activity. In the stable transfection state, conditioned media were collected during 4 days post plating of $2.5 \times 10^{5}$ stably- transfected HepG2 cells and hFIX concentrations were evaluated using ELISA method.

Hek-293T cells were transiently transfected by $2 \mu \mathrm{g}$ of circular constructs mixed with $6 \mu \mathrm{L}$ of FuGene-6. Following $6 \mathrm{~h}$ of post transfection, the supernatants were exchanged by fresh conditioned media containing $1 \mu \mathrm{g} . \mathrm{mL}^{-1}$ vitamin $\mathrm{K} 1$. The supernatants were collected during 3 days post transfection for evaluation of hFIX.

\subsection{Evaluation of hFIX Biological Activity}

The activity of hFIX was determined by one-stage clotting assay as described previously (17). In brief, $100 \mu \mathrm{L}$ of human plasma depleted FIX was mixed with $100 \mu \mathrm{L}$ of aPTT reagent and $100 \mu \mathrm{L}$ of the medium and incubated at $37^{\circ} \mathrm{C}$ for 3 minutes. Next, $100 \mu \mathrm{L}$ of calcium chloride solution $(25 \mathrm{mM})$ was added and coagulation time was recorded. The normal concentration of hFIX in plasma was considered as one unit (21).

pCMV.FIX
prABE/CMV.FIX
pCMV.FIX-I
prABE/CMV.FIX-I
pCMV.FIX-II
prABE/CMV.FIX-II
pCMV.FIX-I,II
prABE/CMV.FIX-I,II

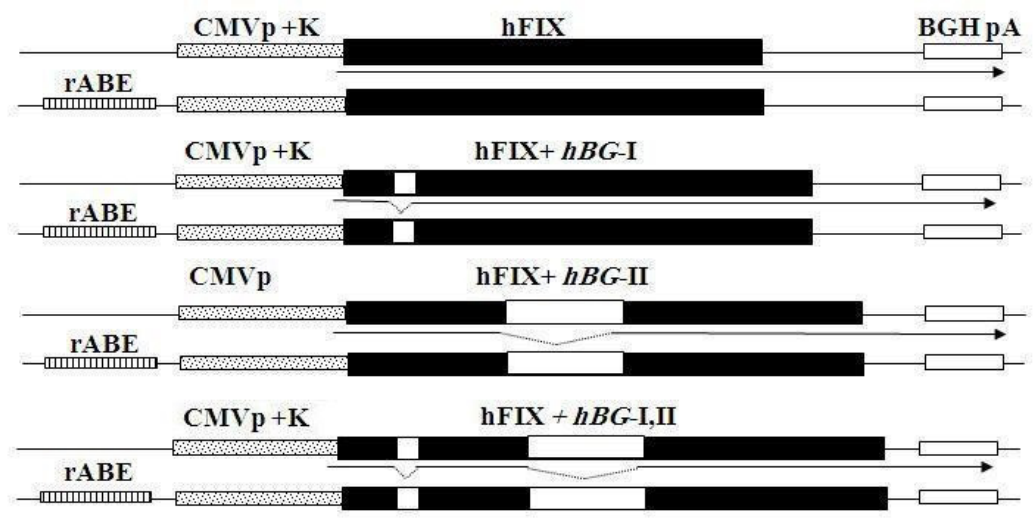

Figure 1. The schematic view of the constructed hFIX expression cassettes in the pcDNA3.1. Names of the plasmids carrying the expression cassettes are indicated on the left. CMVp: cytomegalovirus enhancer/promoter, K: Kozak sequence, $r A B E$ : rat aldolase $\mathrm{B}$ intronic enhancer, $\mathrm{BGH} \mathrm{pA}$ : Bovine growth hormone polyadenylation signal sequence. $h B G$ intron-I (130 bp) which are located between exons 1 and 2 of the hFIX gene. $h B G$ intron-II ( 850 bp) is located between exons 2 and 3 of the hFIX gene and $h B G$ intron-I \& II (130 bp \& $850 \mathrm{bp}$ ) that are located between exons I and II as well as between the exons II and III of the hFIX gene, respectively. The splicing donor and acceptor sequences of the $h B G$ introns were conserved. 


\subsection{Measurement of hFIX Concentration}

Monoclonal antibody against hFIX produced in mouse as a primary antibody and anti-hFIX antibody (produced in goat)-HRP as a secondary antibody were used. The substrate, tetramethyl benzidine (TMB) was finally added and the optical density values were recorded by an ELISA reader. The standard curve was used to calculate hFIX level in the cultured media

\subsection{Evaluation of the Intracellular hFIX Protein Level} Lysate of stably-transfected HepG2 cells were prepared as described earlier (20) and the obtained supernatants were subjected to the Bradford method for determining the total protein levels. Next, hFIX levels in the supernatants were evaluated by ELISA as mentioned above and normalized to the total protein.

\subsection{RNA Préparation and RT-PCR}

To amplify, a preselected region of the $h F I X$ coding sequence, RNAs were isolated from all samples using RNA isolation kit (Roche, Germany) and the cDNA was synthesized according to the manufacturer protocol. Thereafter, the generated cDNA was subjected to the PCR method using specific primers (Table 1). RT-PCRs were performed using specific primer pairs for the human $\beta$-actin (Table 1) as controls. The ratios of the mature hFIX mRNAs and $\beta$-actin band densities were calculated using gel documentation (Gel Logic pro 212 Imaging system, USA).

\subsection{Statistical Analyses}

Statistical analyses were performed by Tukey post-hoc test using SPSS 11.5 software (Chicago, IL, USA). Variables were described by mean $\pm \mathrm{SD}$. $P$ values $<$ 0.05 were considered as statistically significant.

\section{Results}

\subsection{A computer Based Protein-DNA Interactions Analysis}

Analysis of the intronic enhancer sequence $(+1916$ to $+2329 \mathrm{nt})$ of the $r A B$ revealed several motifs distributed throughout the $r A B \mathrm{E}$. In total, 216 transcription factor binding sites were detected within the $r A B E$ (Table 2). In this regard, analysis of the $r A B E$ sequence has provided the relevant information indicating the potential binding sites for liverenriched nuclear factors such as HNF1 (Alpha, Beta), HNF4 (Alpha) as well as CAAT/enhancer binding protein (C/EBP). Our in-silico findings encouraged us to do further studies about the effects of this intronderived fragment on the expression of different hFIX minigenes in the HepG2 cells.

\subsection{Evaluation of the Secreted hFIX From \\ Bioengineered Hep G2 Cells}

To evaluate activity of hFIX expressed from bioengineered HepG2 cells, cell culture medium was collected after $48 \mathrm{~h}$ transfection and was subjected to

Table 2. Putative binding sites for known transcription factors on the $r A B E$ sequence.

\begin{tabular}{lcccccl}
\hline & \multicolumn{2}{c}{ Binding site } & & & \\
\cline { 2 - 3 } Transcription factor & From & To & Strand & Matrix similarity & Sequence \\
\hline Hepatic Nuclear Factor 1 & 57 & 73 & $(-)$ & 0.853 & tGTTAgtctttctaaac \\
TATA-binding protein & 73 & 89 & $(-)$ & 0.866 & acagcaaTATAttatgt \\
TATA-binding protein & 121 & 137 & $(-)$ & 0.840 & tctgtaaTAAAtattta \\
Hepatic Nuclear Factor 4 Alpha & 228 & 252 & $(+)$ & 0.878 & tttaaaggagtaAAGTtcattattg \\
Hepatic Nuclear Factor 1 Alpha & 244 & 260 & $(+)$ & 0.844 & tcattattGTTAagtat \\
Hepatic Nuclear Factor 1 Beta & 249 & 265 & $(-)$ & 0.838 & ctggaataCTTAacaat \\
Hepatic Nuclear Factor 1 & 251 & 267 & $(+)$ & 0.807 & tGTTAagtattccaggc \\
Hepatic Nuclear Factor 1 & 307 & 323 & $(-)$ & 0.891 & agtTAATcataaaacag \\
Hepatic Nuclear Factor 1 Alpha & 314 & 330 & $(-)$ & 0.904 & gcccctcaGTTAatcat \\
CCAAT/enhancer binding protein & 336 & 356 & $(+)$ & 0.799 & ttgtGCTGatgtggtacagac \\
Hepatic Nuclear Factor 4 & 347 & 371 & $(-)$ & 0.874 & acaaagggactAAAGgtctgtacca \\
Hepatic Nuclear Factor 1 & 367 & 383 & $(-)$ & 0.776 & agtTAAActtctacaaa \\
Hepatic Nuclear Factor 1 Alpha & 374 & 390 & $(-)$ & 0.856 & tacaggaaGTTAaactt \\
\hline
\end{tabular}

The rABE sequence retrieved from Ensembl. Putative binding sites for known transcription factors identified by computer search are colored. The rABE sequence was arbitrarily numbered from 1 to 412 . A total of 216 transcription factor binding motifs were found on the rABE sequence which only 13 of them related to the hepatic transcription binding sites are shown. 
Table 3. Clotting activity of hFIX in the supernatant of HepG2 cells transfected with different recombinant plasmids on the second day of post-transfection.

\begin{tabular}{|c|c|}
\hline Recombinant plasmids & Clotting activity (mU.mL $\mathbf{m}^{-1} / 10^{6}$ cells) \\
\hline pCMV.FIX & $* 41 \pm 0.7$ \\
\hline prABE/CMV.FIX & ND \\
\hline pCMV.FIX-I & ${ }^{*} 17 \pm 0.4$ \\
\hline prABE/CMV.FIX-I & ND \\
\hline pCMV.FIX-II & 0 \\
\hline prABE/CMV.FIX-II & 0 \\
\hline pCMV.FIX-I,II & ${ }^{*} 11 \pm 0.5$ \\
\hline prABE/CMV.FIX-I,II & ND \\
\hline
\end{tabular}

HepG2 cells were transfected by $2 \mu \mathrm{g}$ of the circular plasmids and after 48 $\mathrm{h}$ post-transfection, the conditioned medium was collected for evaluation of hFIX biological activity. The results represent the mean \pm SD from two independent experiments. Detectable biological activity of hFIX was not identified in the cultured media of non-transfected cells (data not shown). Asterisk indicates sample that is significantly different $(p<0.05)$ compared to other samples using analysis of variance. ND: not determined.

the one-stage clotting assay. Based on data, $\mathrm{h} B G$ intronII containing plasmid couldn't induce functional hFIX protein secretion (Table 3 ).

We also evaluated hFIX protein level in culture media. For this purpose, The stably-transfected colonies in the selective media were pooled, cultured, and used as representatives of the constructs during expression analysis. In the first step, the representative cellpools were grown up to $50 \%$ confluency before their conditioned media being examined every 24 hours for the next 4 days for the presence of hFIX, by ELISA.
A comparative analysis of the transfected cells with different vectors showed that, the maximum levels of hFIX happen for the $\mathrm{p} r A B \mathrm{E} / \mathrm{CMV}$.FIX construct during experiments (Table 4). When the secretion level on the fourth day was normalized based on the $10^{6}$ cells, the secretion level of hFIX has resulted in 3 folds increase as compared with that of parental CMV-regulated construct (Fig. 2). Next, we studied the effects of the $h B G$ intronic sequences on the hFIX production from HepG2 cells. In this regard, evaluation of the hFIX level in the culture media showed that the first $h B G$ intron is more effective than the second one and the presence of the first $h B G$ intron along with the second one has more effective than either intron I or II alone for secretion of hFIX from HepG2 cells.

On the fourth day, the $r A B E$ has induced 1.3-fold increase in the hFIX level, when it was combined with the $C M V p$ in intron-I containing construct. At the same conditions, $r A B E$ has increased the hFIX levels by nearly 2 folds increase, when it was combined with the CMVp in the intron-II containing construct. Additionally, a stronger activity of the chimeric $r A B \mathrm{E} /$ CMVp compared to CMVp alone in the intron-I and II containing constructs in the hFIX expression was also observed (Fig. 2, Table 4). Based on these data, $r A B \mathrm{E}$ as a liver-specific enhancer is an attractive candidate element to increase hFIX gene expression in hepatocytes.

\subsection{Evaluation of the Expressed hFIX Within the Bioengineered Hepatic Cell Line}

The maximum intracellularhFIX levels were obtained from the $\mathrm{p} A B \mathrm{E} / \mathrm{CMV}$.FIX as well as pCMV.FIX constructs,

Table 4. Determination of the hFIX level in the supernatant of stably-transfected HepG2 cells at various culture durations, based on ELISA.

\begin{tabular}{|c|c|c|c|c|}
\hline \multirow[b]{2}{*}{ Construct } & \multicolumn{4}{|c|}{ ELISA (ng.mL ${ }^{-1}$ ) } \\
\hline & Day 1 & Day 2 & Day 3 & Day 4 \\
\hline pCMV.FIX & $\mathrm{a} 3.3 \pm 0.2$ & $\mathrm{a} 8.5 \pm 0.3$ & ${ }^{\mathrm{a}} 13.5 \pm 4.9$ & a $26.5 \pm 4.9$ \\
\hline prABE/CMV.FIX & ${ }^{\mathrm{b}} 15 \pm 1$ & ${ }^{\mathrm{b}} 30 \pm 4.9$ & ${ }^{\mathrm{b}} 55 \pm 7.1$ & b78.5 \pm 3.5 \\
\hline pCMV.FIX-I & ${ }^{\mathrm{c}} 1.4 \pm 0.4$ & ${ }^{\mathrm{c}} 1.7 \pm 0.4$ & $c^{c} 2 \pm 0.2$ & $\mathrm{c} 5.6 \pm 0.6$ \\
\hline prABE/CMV.FIX-I & $\mathrm{a}, \mathrm{d} 2.6 \pm 0.6$ & $\mathrm{~d} 2.5 \pm 0.4$ & $\mathrm{~d} 3.4 \pm 0.3$ & $\mathrm{~d} 7.8 \pm 1.1$ \\
\hline pCMV.FIX-II & ${ }^{\mathrm{c}} 1.6 \pm 0.4$ & $\mathrm{c}, \mathrm{d} 1.9 \pm 0.2$ & ${ }^{\mathrm{c}, \mathrm{d}} 2.7 \pm 1$ & $\mathrm{e} 4.3 \pm 0.4$ \\
\hline prABE/CMV.FIX-II & $\mathrm{a}, \mathrm{d} 3.3 \pm 1.3$ & $\mathrm{e} 3.9 \pm 0.7$ & ${ }^{\mathrm{e}} 6.7 \pm 1.3$ & $\mathrm{~d} 7.9 \pm 0.4$ \\
\hline pCMV.FIX-I,II & ${ }^{\mathrm{c}} 1.7 \pm 0.1$ & ${ }^{\mathrm{c}} 1.1 \pm 0.1$ & ${ }^{\mathrm{c}} 1.4 \pm 0.6$ & ${ }^{\mathrm{c}, \mathrm{d}} 6.3 \pm 1.3$ \\
\hline prABE/CMV.FIX-I,II & $\mathrm{d} 2.3 \pm 0.4$ & $\mathrm{c}, \mathrm{d} 1.9 \pm 0.1$ & ${ }^{\mathrm{f}} 4.1 \pm 0.3$ & f $9.8 \pm 0.3$ \\
\hline
\end{tabular}

The results represent the mean \pm SD from two independent experiments with duplicate assays. Detectable level of hFIX was not identified in the cultured media of non-transfected cells (Data not shown). Different letters indicate that there is a significant difference between two samples at the same time point $(p<0.05)$. 


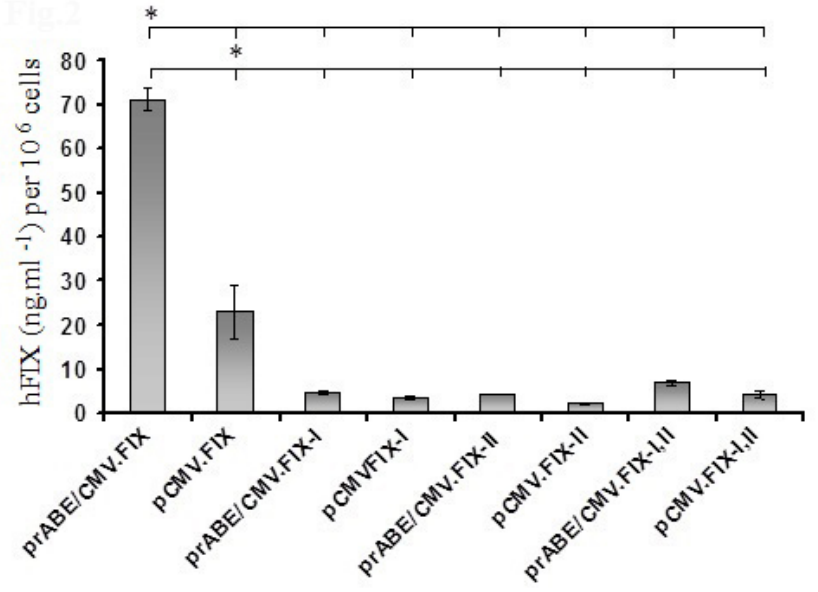

Figure 2. Evaluation of the hFIX levels in the supernatant of the stably-transfected HepG2 cells using ELISA on the $4^{\text {th }}$ day of post-plating. The results are represented as the mean of hFIX secretion of the two independent cell-pools with duplicate assays. ${ }^{*} p<0.05$.

and were measured to be 74.1 and 51.6 ng.mg ${ }^{-1}$ protein, respectively. The lowest level of hFIX within the cells was obtained from the pCMV.FIX-I, II containing cells (Fig. 3). The $r A B \mathrm{E}$ in combination with CMVp in the context of intron-less plasmid was seen to increase dramatically the total expression ofhFIX protein, ranging from 2.3 to 40 folds increase compared to the other constructs and $\mathrm{p} r A B \mathrm{E} / \mathrm{CMV}$.FIX, pCMV.FIX as well as $\mathrm{p} r A B \mathrm{E} / \mathrm{CMV}$. FIX-I have the highest influence on the hFIX protein level in the HepG2 cell line respectively (Table 5).

4.4. FIX Secretion Efficiency in the Recombinant HepG2 Cell Line

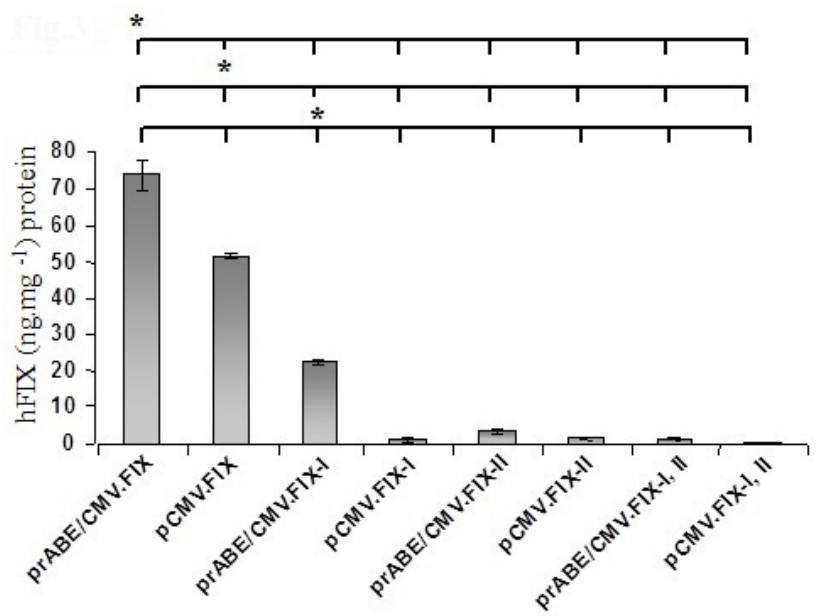

Figure 3. Evaluation of the intracellular hFIX levels in the stably-transfected HepG2 cells using ELISA on the $4^{\text {th }}$ day of post-plating. The results represent the mean hFIX levels of the two independent experiments with the duplicate assays. ${ }^{*} p<0.05$.

FIX secretion efficiency was calculated as described previously (7). In this study, pCMV.FIX-I,II, prABE/ CMV.FIX, I,II, and pCMV.FIX-I had the highest hFIX secretion efficiency in the HepG2 cells compared to the other constructs (Fig. 4).

4.5. Analysis of the Intron Removal from the hFIX Transcripts in Stably-Transfected Hepatic Cells

Total RNAs were isolated from all samples followed by cDNA synthesis and RT-PCR. Our RT-PCR results showed that $r A B \mathrm{E}$ in the context of intron-less plasmid has induced the highest level of mature hFIX mRNAs

Table 5. Evaluation of engineered plasmid vectors for total expression of hFIX protein (Extracellular + Intracellular) in HepG2 cells.

\begin{tabular}{lcccccccc}
\hline & \multicolumn{7}{c}{ Fold hFIX expression (Extracellular + Intracellular) } \\
\cline { 2 - 9 } Plasmid & $\begin{array}{c}\text { prABE/CMV } \\
\text {.FIX }\end{array}$ & $\begin{array}{c}\text { pCMV } \\
\text {.FIX }\end{array}$ & $\begin{array}{c}\text { prABE/CMV } \\
\text {.FIX-I }\end{array}$ & $\begin{array}{c}\text { pCMV } \\
\text {.FIX-I }\end{array}$ & $\begin{array}{c}\text { prABE/CMV } \\
\text {.FIX-II }\end{array}$ & $\begin{array}{c}\text { pCMV } \\
\text {.FIX-II }\end{array}$ & $\begin{array}{c}\text { prABE/CMV } \\
\text {.FIX-I, II }\end{array}$ & pCMV \\
.FIX-I, II \\
\hline prABE/CMV.FIX vs. & 1 & $2.3^{*}$ & $8.1^{*}$ & $29.8^{*}$ & $20.4^{*}$ & $40^{*}$ & $15.1^{*}$ & $24.7^{*}$ \\
pCMV.FIX vs. & $0.4^{*}$ & 1 & $3.8^{*}$ & $13.1^{*}$ & $8.9^{*}$ & $17.6^{*}$ & $6.6^{*}$ & $10.8^{*}$ \\
prABE/CMV.FIX-I vs. & $0.03^{*}$ & $0.08^{*}$ & 1 & $3.7^{*}$ & $2.5^{*}$ & $5^{*}$ & $1.9^{*}$ & $3.1^{*}$ \\
pCMV.FIX-I vs. & $0.03^{*}$ & $0.08^{*}$ & $0.3^{*}$ & 1 & 0.7 & 1.4 & 0.5 & 0.8 \\
prABE/CMV.FIX-II vs. & $0.05^{*}$ & $0.1^{*}$ & $0.4^{*}$ & 1.5 & 1 & $2^{*}$ & 0.7 & 1.2 \\
pCMV.FIX-II vs. & $0.02^{*}$ & $0.06^{*}$ & $0.2^{*}$ & 0.7 & 0.5 & 1 & $0.4^{*}$ & $0.06^{*}$ \\
prABE/CMV.FIX-I,II vs. & $0.07^{*}$ & $0.2^{*}$ & 0.5 & $2.3^{*}$ & 1.4 & $2.7^{*}$ & 1 & $1.6^{*}$ \\
pCMV/FIX-I,II vs. & $0.04^{*}$ & $0.09^{*}$ & $0.3^{*}$ & 1.2 & 0.8 & $1.6^{*}$ & 0.6 & 1 \\
\hline
\end{tabular}

Total hFIX protein levels produced by different vectors were calculated using ELISA method and normalized by the cell numbers. To compare expression levels of hFIX by different vectors, hFIX protein level produced by a vector divided to the hFIX protein levels produced by other vectors. All experiments were repeated twice using duplicate assays and the results were presented as the mean. Asterisk indicates the difference between two different constructs for expression of the hFIX $(p<0.05)$. 


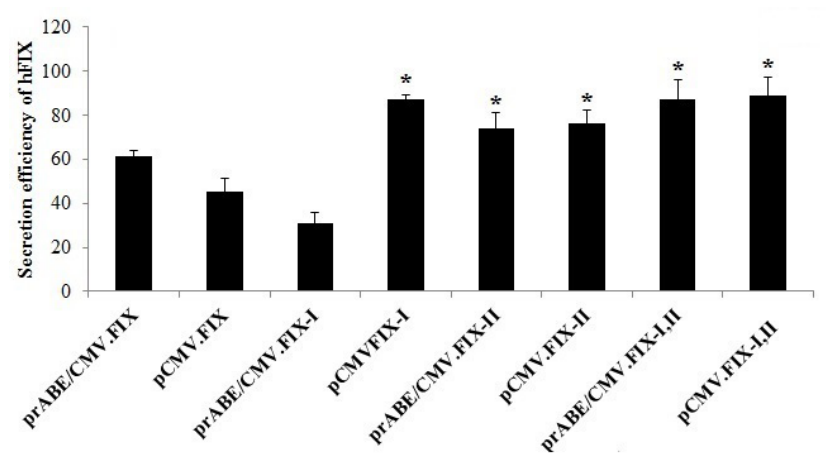

Figure 4. The secretion efficiency of the hFIX on the fourth day of post-transfection in the genetically modified HepG2 cells vs the parental intron-less construct. The results represent the mean \pm SD from the two independent experiments and each assay in a duplicate manner.

compared to other constructs. Evaluation of the intron removal from pre-mRNAs generated from the introncontaining constructs also showed that in spite of $h B G$ intron-II containing construct, the cells transfected with $h B G$ intron-I containing construct are able to generate mature hFIX mRNAs. Interestingly, the presence of $h B G$ intron-I along with the intron II in the construct provides a correct splicing in hFIX pre-mRNAs and generates the higher level of mature hFIX mRNAs than either $h B G$ intron-I or II containing construct alone (Fig. 5).

\subsection{Expression of hFIX from the Transiently- Transfected Kidney Cells}

To investigate the $h B G$ introns functions on the secretion of hFIX in another cell line, pCMV.FIX, pCMV.FIX-I, pCMV.FIX-II, and pCMV.FIX-I,II plasmids were transiently transfected into Hek-293T cells and the hFIX level in the cultured media were measured during 3 days of cultivation (Fig. 6). Based on data, the highest expression levels were obtained from the pCMV.FIX and pCMV.FIX-I constructs, respectively. When the cell numbers were normalized per $10^{6}$ cells, the hFIX levels in the pCMV.FIX, pCMV.FIX-I, pCMV.FIXII, and pCMV.FIX-I, II plasmids were measured to be 43.6, 29.3, 16.6 and $13.4 \mathrm{ng} . \mathrm{mL}^{-1}$, respectively.

\subsection{Analysis of the Intron Removal from the $h F I X$ Transcripts in Transiently-Transfected Kidney Cells}

To investigate the possible function of the cell type on the proper intron-splicing of a transcript precursor, Hek-293T cells were transiently transfected with the bioengineered plasmids and after $48 \mathrm{~h}$ post transfection, the total RNAs were isolated from all samples and
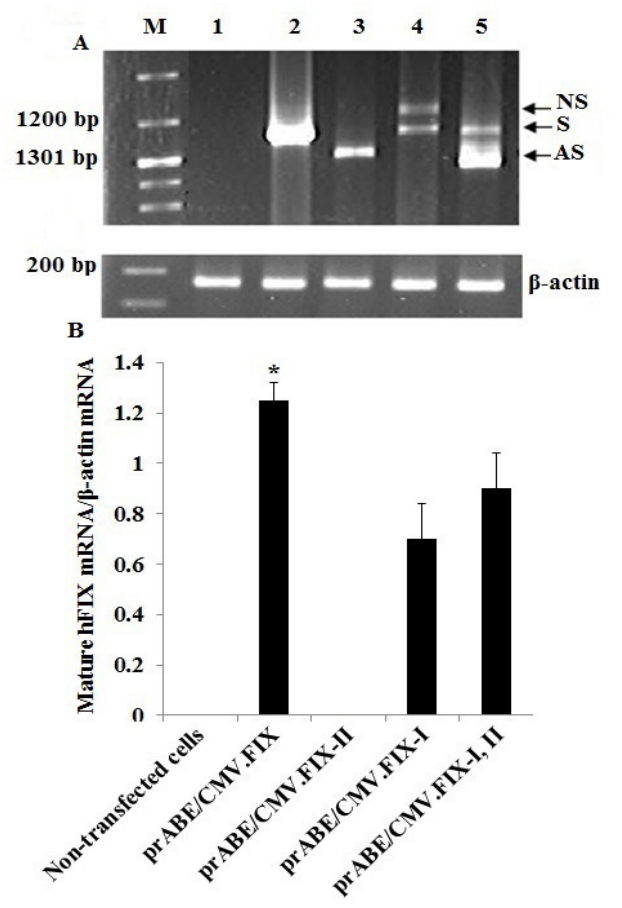

Figure 5. Evaluation of the hFIX expression through application of RT-PCR in the transfected HepG2 cells. Panel A: Lane M: DNA size marker, NS: Non-spliced product, S: Spliced product (mature hFIX mRNAs), AS: Aberrant spliced product. Non-transfected (Lane 1) and HepG2 cells transfected with the prABE/CMV.FIX-II construct (Lane 3) did not show detectable hFIX mRNAs. Panel B: Histogram presentation of hFIX mRNA expression from different constructs. Asterisks indicate a significant difference between tested samples $\left({ }^{*} p<0.05\right)$.

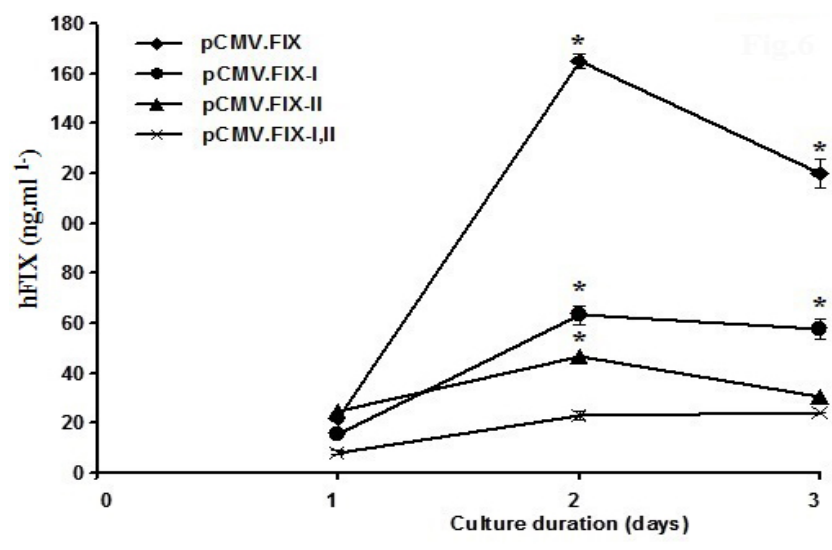

Figure 6. Determination of the hFIX level in the cultured media of the Hek-293T cells at various culture durations based on ELISA. The results represent the mean hFIX secretion of the two independent experiments with duplicate of each assay. A detectable level of hFIX was not identified in the culture supernatant of the non-transfected Hek-293T cells (data are not shown). Asterisks indicate a significant difference in the hFIX expression between tested samples for the same time points. $\left({ }^{*} p<0.05\right)$. 

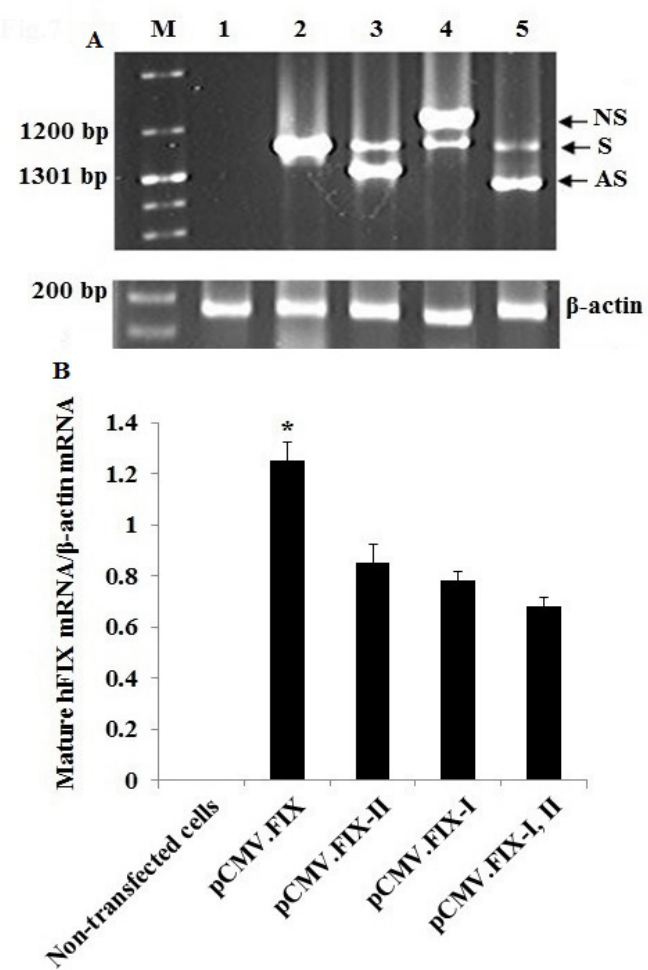

Figure 7. Detection of hFIX mRNA levels using RT-PCR in the Hek-293T cells. Panel A: Lane M: DNA size marker, NS: Non-spliced product, S: Spliced product (mature hFIX mRNAs), AS: Aberrant spliced product. Non-transfected cells (Lane 1) did not show a detectable hFIX mRNA change. Panel B: Histogram presentation of hFIX expression from different constructs. Asterisks indicate a significant difference between tested samples ( ${ }^{*} p<0.05$ ).

were subjected to the RT-PCR. In Hek-293T cells, all constructs were able to generate mature hFIX mRNAs. The maximum and minimum levels of the mature hFIX mRNAs were obtained from the intron-less and $h B G$ intron-I,II containing constructs, respectively. Interestingly, mature hFIX mRNAs were generated from the $h B G$ intron-II containing construct, whereas it was not detect from the same construct in the HepG2 cells (Fig. 5 and 7).

\section{Discussion}

The use of strong viral or tissue-specific promoters and enhancers, insertion of introns upstream of cDNAs or into various cDNAs, and optimization of the nucleotide context around the initiation ATG codon (Kozak element) have been used to improve gene expression on transcriptional, post-transcriptional, and translational levels in-vitro or in-vivo (22-25). Promising results might be obtained if we consider all of these approaches together in a systematic study, in this context.

Plasmid constructs are less toxic, with low potential risk of random integration into chromosomal DNA, easier to prepare with consistent quality in large scale, repeated administration of these vectors, if necessary, and unable to generate harmful replication-competent (26).

One advantage of using stable pools of HepG2 cells over the transient transfection is that a large number of resistant cells constitutively secrete the hFIX protein and could be considered as in-vitro models for tissues transduced by protein expressing vectors such as the ones used in the gene therapy approaches. In this context, the level of hFIX in the culture supernatants and within the recombinant cells have shown different concentrations of the hFIX which suggest differences in the cis-regulatory elements used in each of engineered plasmid results in the differences in the posttranscriptional regulation of the hFIX.

In-silico findings along with the in-vitro experiments have demonstrated the presence of regulatory motifs in the $r A B \mathrm{E}$. These findings are in agreement with the results that have previously been published by Majewski and colleagues who have shown that the first intron of the mammalian genes help transcription, due to the presence of transcription factor binding sites (27). In our study, $r A B E$ in combination with CMVp in intron-less construct has induced the highest level of hFIX expression compared to the other constructs. Consistent with our results, other investigators have also shown that $r A B \mathrm{E}$ is functional in both orientation and in any position, either upstream or downstream to the promoter and is able to stimulate heterologous promoters activity, as well $(28,29)$. Furthermore, in similar study, muscle creatine kinase enhancer, which is active specifically in the muscles, increased the CMVregulated expression of the human and canine FIXs invitro and in-vivo, respectively $(9,30)$.

Our results and those reported by others show that tissue-specific enhancers are responsible for the increase in the activity of CMVp. Such a chimeric regulatory sequence might be able to prevent shutdown of the CMVp in-vivo as it was demonstrated previously (30).

Introduction of the intronic sequences inside the transgene cDNA may provide a higher expression level of a therapeutic gene, as well. In this regard, enhancerlike activity of the $h B G$ intron-II and intron-I,II inside the coding region of the hFIX on the hFIX level have been documented $(7,17)$. However, in our hepatocytebased expression system, $h B G$ introns have negative effects on the expression of hFIX compared to the intron-less construct.

A major problem associated with the secretion 
of recombinant hFIX is the poor yield of functional gamma-carboxylated proteins from transfected nonhepatic cells. Based on coagulation assays, hFIX secreted from transfected HepG2 cells had biological activity, suggesting the presence of appropriate posttranslational modifications in the hFIX.

In other instances, it has been shown that heterologous introns are not spliced as efficiently as the natural introns $(31,18)$. In our study, the heterologous $h B G$ intronic sequences in their identical positions within the $h F I X$-cDNA are not completely spliced that can probably explain the low level of hFIX in the $h B G$ intron-containing $h F I X$ minigenes in the HepG2 and Hek-293T cell lines. Interestingly, despite HepG2, Hek$293 \mathrm{~T}$ cells were able to generate mature hFIX mRNAs from the $h B G$ intron-II containing construct. At this stage, the type of cell lineage and exon-intron structure may have important roles in the correct splicing of an intron from a transcript precursor.

In our experiments, mature hFIX mRNAs were not generated from the $h B G$ intron-II containing plasmids in the HepG2 cells. This result demonstrates that why HepG2 cells carrying $h B G$ intron-II containing construct couldn't secret functional hFIX to the conditioned medium.

Our results have demonstrated that the presence of the $r A B \mathrm{E}$ in the intron-less construct has strongly increased hFIX mRNA level, suggesting a cooperation of $r A B \mathrm{E}$ with the strong $\mathrm{CMVp}$ for the transcription of hFIX. Furthermore, the presence of both $h B G$ introns in the vector plasmid has induced a higher mature hFIX mRNA level than either $h B G$ intron-I or II construct alone in the HepG2 expression system which suggest the cooperation of the two $h B G$ introns to enhance the mature hFIX mRNA level.

Based on our data, $\mathrm{p} r A B \mathrm{E} / \mathrm{CMV}$.FIX construct has induced higher hFIX secretion which was accompanied by a higher hFIX protein expression within the cells. In this regard, different investigators have shown that the recombinant cells with higher expression of recombinant protein were susceptible to accumulation of more protein than lower expression ones (31-33). Considering the cellular chaperones and reduction of possible hFIX binding to these proteins may cooperate to improve hFIX secretion, accordingly.

In conclusion, the present study is the first report on the effects of different heterologous intron-derived cisregulatory elements intra and extra coding region of the $h F I X$ gene in the hepatic and embryonic cell lines. The synergistic effect on the hFIX expression achieved by a combination of the CMVp with the liver-specific $r A B \mathrm{E}$ would be a useful approach in the future designs of the expression cassettes. In this regard, addition of the multiple copies of the $r A B E$ element upstream of the CMVp may synergistically increase hFIX expression in-vitro and/or in-vivo.

\section{References}

1. Peyvandi F, Garagiola I, Young G. The past and future of haemophilia: diagnosis, treatments, and its complications. Lancet. 2016;388(10040):187-97. doi: 10.1016/S01406736(15)01123-X.

2. Bigger BW, Siapati EK, Mistry A, Waddington SN, Nivsarkar MS, Jacobs L, et al. Permanent partial phenotypic correction and tolerance in a mouse model of hemophilia B by stem cell gene delivery of human factor IX. Gene Ther. 2006;13(2):117126. doi: $10.1038 /$ sj.gt.3302638

3. Chang AH, Stephan MT, Sadelain M. Stem cell-derived erythroid cells mediate long-term systemic protein delivery. Nat Biotechnol. 2006;24(8):1017-1021. doi:10.1038/nbt1227

4. George LA, Fogarty PF. Gene therapy for hemophilia: past, present and future. Semin Hematol. 2016:53(1):46-54. doi: 10.1053/j.seminhematol.2015.10.002

5. Tatsumi K, Ohashi K, Kataoka M, Tateno C, Shibata M, Naka H. Successful in vivo propagation of factor IX-producing hepatocytes in mice: potential for cell-based therapy in haemophilia B. Thromb Haemost. 2008;99(5):883-891.doi: 10.1160/TH07-09-0559

6. Krebsbach PH, Zhang K, Malik AK, Kurachi K. Bone marrow stromal cells as a genetic platform for systemic delivery of therapeutic proteins in vivo: human factor IX model. $J$ Gene Med. 2003;5(1):7-11. doi: 10.1002/jgm.292

7. Sam MR, Azadbakhsh AS, Farokhi F, Rezazadeh K, Sam $\mathrm{S}$, Zomorodipour A. Genetic modification of bone-marrow mesenchymal stem cells and hematopoietic cells with human coagulation factor IX-expressing plasmids. Biologicals. 2016;44(3):170-177. doi: 10.1016/j.biologicals.2016.01.002

8. Hosseini SJ, Zomorodipour A, Jalal R, Sabouni F, Ataei F. A study of the expression of functional human coagulation factor IX in keratinocytes using a nonviral vector regulated by K14 promoter. Appl Biochem Biotechnol. 2010;162(6):1599-1611. doi: 10.1007/s12010-010-8941-0

9. Dai Y, Roman M, Naviaux RK, Verma IM. Gene therapy via primary myoblasts: long-term expression of factor IX protein following transplantation in vivo. Proc Natl Acad Sci USA. 1992;89(22):10892-10895.

10. Yao SN, Wilson JM, Nabel EG, Kurachi S, Hachiya HL, Kurachi $\mathrm{K}$. Expression of human factor IX in rat capillary endothelial cells: toward somatic gene therapy for hemophilia B. Proc Natl Acad Sci USA. 1991;88(18):8101-8105.

11. Armentano D, Thompson AR, Darlington G, Woo SL. Expression of human factor IX in rabbit hepatocytes by retrovirus-mediated gene transfer: potential for gene therapy of hemophilia B. Proc Natl Acad Sci USA. 1990;87(16):6141-6145.

12. Dobrzynski E, Herzog RW. Tolerance induction by viral in vivo gene transfer. Clin Med Res. 2005;3(4):234-240. doi: 10.3121/ cmr.3.4.234

13. Okkema PG, Harrison SW, Plunger V, AryanaA, Fire A. Sequence requirements for myosin gene expression and regulation in Caenorhabditis elegans. Genetics. 1993;135(2):385-404.

14. Lu S, Cullen BR. Analysis of the stimulatory effect of splicing on mRNA production and utilization in mammalian cells. $R N A$. 
2003;9(5):618-630. doi:10.1261/rna.5260303

15. Jonsson JJ, Foresman MD, Wilson N, Aryana A, Fire A. Intron requirement for expression of the human purine nucleoside phosphorylase gene. Nucleic Acids Res. 1992:20(12):31913198. doi:10.1093/nar/20.12.3191

16. Neel H, Weil D, Giansante C, Dautry F. In vivo cooperation between introns during pre-mRNA processing. Genes Dev. 1993;7(11):2194-2195. doi:10.1101/gad.7.11.2194

17. Haddad-Mashadrizeh A, Zomorodipour A, Izadpanah M, Sam MR, Ataei F, Sabouni F.A systematic study of the function of the human beta-globin introns on the expression of the human coagulation factor IX in cultured Chinese hamster ovary cells. $J$ Gene Med. 2009;11(10):941-950. doi: 10.1002/jgm.1367

18. Noe V, MacKenzie S, Ciudad CJ. An intron is required for dihydrofolatereductase protein stability. J Biol Chem. 2003;278(40):38292-38300. doi: 10.1074/jbc.M212746200

19. Fair DS, Bahnak BR. Human hepatoma cells secrete single chain factor $\mathrm{X}$, prothrombin, and antithrombin III. Blood.1984;64(1):194-204.

20. Sam MR, Zomorodipour A, Shokrgozar MA, Ataei F, HaddadMashadrizeh A, Amanzadeh A. Enhancement of the human factor IX expression, mediated by an intron derived fragment from the rat aldolase B gene in cultured hepatoma cells. Biotechnol Lett. 2010;32(10):1385-1392. doi: 10.1007/s10529010-0321-x.

21. Page SM, Brownlee GG. An ex vivo keratinocyte model for genetherapy of hemophilia B. J Invest Dermatol. 1997;109(2):139-145. doi: 10.1111/1523-1747.ep12319194

22. Jallat S, Perraud F, Dalemans W, Balland A, Dieterle A, Faure $\mathrm{T}$, et al. Characterization of recombinant human factor IX expressed in transgenic mice and in derived trans-immortalized hepatic cell lines. EMBO J. 1990;9(10):3295-3301.

23. Brinster RL, Allen JM, Behringer RR, Gelinas RE, Palmiter $\mathrm{RD}$. Introns increase transcriptional efficiency in transgenic mice. Proc Natl Acad Sci USA.1988;85(3):836-840.
24. Furger A, O’Sullivan JM, Binnie A, Lee BA, Proudfoot NJ. Promoter proximal splice sites enhance transcription. Genes Dev. 2002;16(21):5795-5799. doi: 10.1101/gad.983602

25. Le Hir H, Nott A, Moore MJ. How introns influence and enhance eukaryotic gene expression. Trends Biochem Sci. 2003;28(4):215-220. doi: 10.1016/S0968-0004(03)00052-5

26. Miao CH, Thompson AR, Loeb K, Ye X. Long-term and therapeutic-level hepatic gene expression of human factor IX after naked plasmid transfer in vivo. Mol Ther. 2001;3(6):947957.doi: $10.1006 /$ mthe.2001.0333

27. Majewski J, Ott J. Distribution and characterization of regulatory elements in the human genome. Genome Res. 2002;12(12):1827-1836. doi: 10.1101/gr.606402

28. Gregori C, Porteu A, Lopez S, Kahn A, Pichard AL. Characterization of the aldolase B intronic enhancer.J Biol Chem. 1998;273(39):25237-25243. doi: 10.1074/jbc.273.39.25237

29. Sabourin JC, Kern AS, Gregori C, Porteu A, Cywiner C, Châtelet FP, et al. An intronic enhancer essential for tissuespecific expression of the aldolase B transgenes. $J$ Biol Chem. 1996;271(7):3469-3473. doi: 10.1074/jbc.271.7.3469

30. Hagstrom JN, Couto LB, Scallan C, Burton M, McCleland ML, Fields PA, et al. Improved muscle-derived expression of human coagulation factor IX from a skeletal actin/CMV hybrid enhancer/promoter. Blood. 2000;95(8):2536-2542.

31. Plantier JL, Rodriguez MH, Enjolras N, Attali O, Négrier C. A factor VIII minigene comprising the truncated intron I of factor IX highly improves the in vitro production of factor VIII. Thromb Haemost. 2001;86(2):596-603.

32. 32. Tagliavacca L, Wang Q, Kaufman RJ. ATP-dependent dissociation of non-disulfide-linked aggregates of coagulation factor VIII is a rate-limiting step for secretion. Biochemistry. 2000;39(8):1973-1981. doi: 10.1021/bi991896r

33. Rodriguez MH, Enjolras N, Plantier JL, Réa M, Leboeuf M, Uzan G, et al. Expression of coagulation factor IX in a haematopoietic cell line. Thromb Haemost. 2002;87(3):366373. 